\title{
Habituation and Dishabituation of a Cleaning Reflex in Normal and Mutant Drosophila
}

\author{
Gabriel Corfas and Yadin Dudai \\ Department of Neurobiology, The Weizmann Institute of Science, Rehovot 76100, Israel
}

\begin{abstract}
Upon tactile stimulation of its thoracic bristle(s), Drosophila cleans with a patterned set of leg movements the field covered by the stimulated bristles. We demonstrate that this cleaning reflex undergoes habituation and dishabituation. Repeated monotonous stimulation of the bristles by controlled air puffs leads to decrement, and finally to disappearance, of leg response. Spontaneous recovery of the response takes place in a time-dependent manner. Restoration of response can also be obtained by application of a high-frequency stimulus directed to other bristles. A mutant, rut, which is defective in learning and in adenylate cyclase activity, can habituate and dishabituate, but habituation is abnormally short-lived. As opposed to both nonassociative and associative learning paradigms used in Drosophila to date, the cleaning reflex lends itself to some aspects of cellular analysis, since single sensory neurons that mediate the input and motor neurons that mediate the behavioral output are identifiable. The modified reflex should therefore be useful in establishing the effects of single gene mutations that affect behavioral plasticity on the development and properties of identified neurons that contribute to discrete modifiable behaviors.
\end{abstract}

Studies of simple learning processes have cast light in recent years on elementary cellular and molecular mechanisms used by nervous systems to acquire and retain information (Kandel and Schwartz, 1982; Alkon, 1984; Quinn, 1984; Byrne, 1985; Carew and Sahley, 1986; Dudai, 1986; Goelet et al., 1986). For such studies, relatively simple organisms often offer substantial experimental advantages. For example, mollusks such as Aplysia and Hermissenda are employed to probe the contribution of identified neuronal elements to plastic changes in networks that underlie discrete modifiable reflexes (Kandel and Schwartz, 1982; Alkon, 1984; Byrne, 1985; Goelet et al., 1986). The fruit fly, Drosophila melanogaster, is especially valuable for genetic dissection of learning (Accves-Piña ct al., 1983; Dudai, 1986). Single gene mutations of Drosophila have been identified that reduce learning and memory relatively specifically, and the molecular lesions caused by several such mutations are associated with defective function of second-messenger systems, e.g., the cAMP cascade (Aceves-Piña et al., 1983; Dudai, 1986, 1987).

\footnotetext{
Received Oct. 12, 1987; revised May 23, 1988; accepted May 24, 1988.

This study was supported in part by the US-Israel Binational Science Foundation, Jerusalem, and by the US-Israel Binational Agricultural Research and Development Fund (BARD). We wish to thank V. Krongaus for the use of video recording equipment.

Correspondence should be addressed to Yadin Dudai at the above address. Copyright (C) 1989 Society for Neuroscience $0270-6474 / 89 / 010056-07 \$ 02.00 / 0$
}

Most studies of learning in Drosophila have employed associative learning paradigms, which are either a combination of instrumental conditioning and classical conditioning (Quinn et al., 1974; Dudai, 1977) or classical conditioning (Tully and Quinn, 1985). In such paradigms (see also Menne and Spatz, 1977; Hall, 1984; Mariath, 1985), the fly's behavior is complex, and many sensory and motor systems are involved in the modified response. The neuronal networks underlying these behaviors have not been identified. Certain nonassociative learning paradigms, i.e., habituation and sensitization, have also been described for Drosophila, but again, the behaviors involved are highly complex and identification of neuronal components is as yet infeasible (Fischbach, 1981; Duerr and Quinn, 1982). It should be advantageous to identify in Drosophila relatively simple modifiable reflexes, in which at least part of the neurons that mediate the reflex could be identified. This would potentially pave the way to cellular analysis of the effect of mutations on plasticity. Among the questions that could be approached in such a system would be the effect of single-gene mutations that affect behavioral plasticity on the development, morphology, connectivity, pharmacology, and physiology of identified neurons. The relation between behavioral plasticity and development is of special interest in this context in view of the postulated role of second-messenger cascades in both processes (reviewed in Nestler and Greengard, 1984; Dudai, 1987).

In this paper, we report studies on a cleaning reflex of Drosophila. This is a reflex in which a fly, upon tactile stimulation of its bristle(s), cleans the field covered by the stimulated bristle(s) (Vandervorst and Ghysen, 1980). The cleaning reflex is an especially suitable potential candidate for studies of certain aspects of plasticity, since single identified sensory neurons mediate discrete components of the input (Ghysen, 1980), and motoneurons mediating the behavioral output are identifiable (Power, 1948; Green, 1981). We describe and quantify the reflexive behavior and demonstrate that it is modified by experience. We also show that a mutation that lesions the adenylate cyclase system interferes with behavioral plasticity of the reflex.

\section{Materials and Methods}

Flies. Canton-S (CS) flies were used as wild-type. The mutant rutabaga (rut) is on a CS genetic background (Duerr and Quinn, 1982) and was kept in an homozygous stock. Olfactory classical conditioning assays (Tully and Quinn, 1985) and adenylate cyclase assays (Dudai et al., 1983 ) were performed periodically and concurrently on CS and rut flies, to reassure lack of stock contaminations or accumulation of genetic modifiers. All flies were cultured under standard conditions (Lewis, 1960). Female flies (4-8 d old) were used for experiments. Fly stocks were kept at $24 \pm 1{ }^{\circ} \mathrm{C}, 50-70 \%$ humidity, $12 \mathrm{hr}$ light-12 hr dark cycles. Experiments were performed at $23 \pm 2^{\circ} \mathrm{C}, 50-70 \%$ humidity.

Preparation of flies. Experiments were performed on headless flies. 

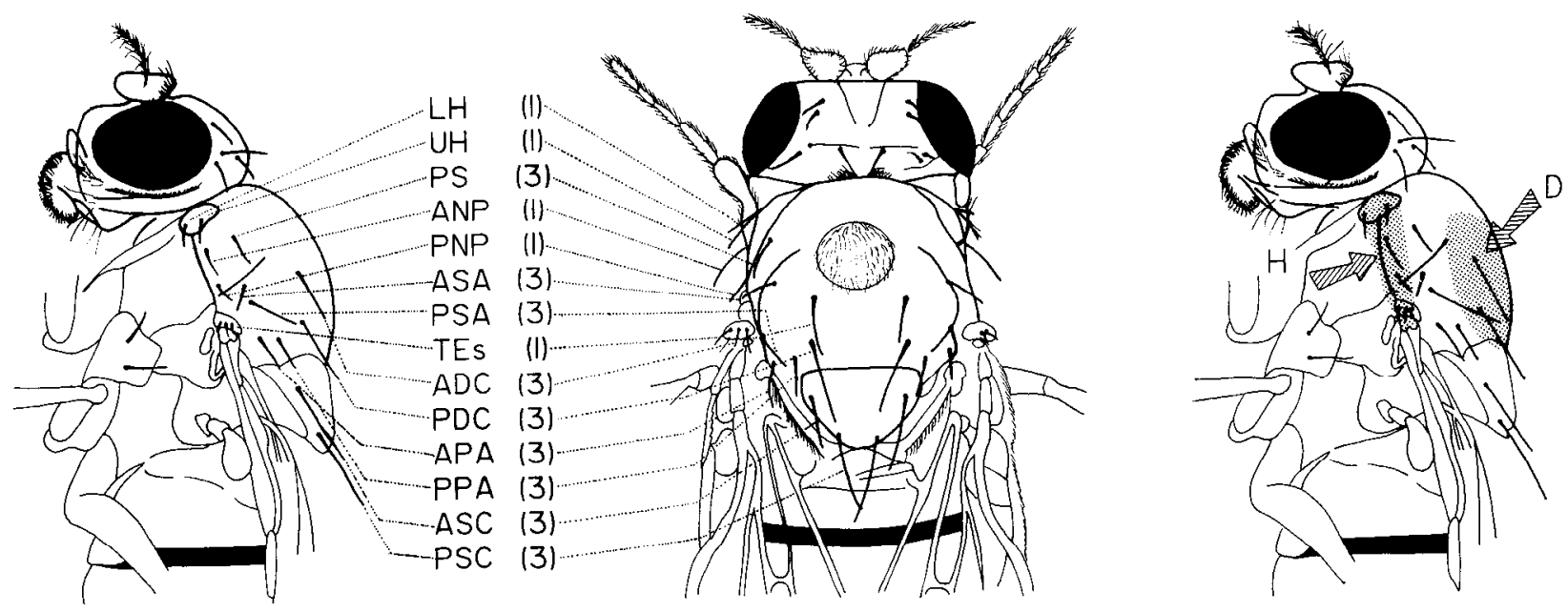

Figure 1. Schematic drawing of lateral and dorsal views of the anterior part of Drosophila, depicting the position of the thoracic macrochaetae. The leg that responds to stimulation of the corresponding bristle is indicated in parentheses. Key: Upper-humeral $(U H)$, lower-humeral $(L H)$, presutural $(P S)$, antcro-noto-plcural $(A N P)$, tegulars $(T E S)$, antero-supra-alar $(A S A)$, postero-supra-alar $(P S A)$, antero-dorso-central $(A D C)$, posterodorso-central $(P D C)$, antero-post-alar $(A P A)$, postero-post-alar $(P P A)$, antero-scutellar $(A S C)$, and postero-scutellar $(P S C)$. The circle in the center of the notum represents a field of microchaetae that cover all the notum but were omitted from the drawing for simplicity. The right-hand figure depicts the areas (stippled) stimulated by the habituating $(H)$ and dishabituating $(D)$ channels (see Materials and Methods and Fig. 3).

Decapitation was performed with iridectomy scissors under light cold anesthesia. Flies were allowed to recover from trauma for $1 \mathrm{hr}$ inside a 35-mm-diameter Petri dish containing a wet piece of cotton. Only flies that were able to stand correctly on all their legs and to move in response to tactile stimuli were used for experiments.

Determination of sensory fields. Flies were fixed on their side by embedding all 3 legs and the ipsilateral wing in modelling clay. The flies were kept in a closed plastic box containing wet Kimwipe paper tissue. Stimulation was delivered manually to the bristle (Fig. 1) with a single human hair under a dissecting microscope. Each bristle was stimulated 20 consecutive times at $10 \mathrm{sec}$ intervals and the number of responses and the leg involved were recorded visually.

Video recording. To follow in detail the movements of the legs during the behavioral response (Fig. 2), video recording was performed using a Panasonic color video camera WV3980e and an AG 6200 VCR connected to a zoom stereoscope (M8, Wild, Heerbrugg). The flies were mounted on their side on modelling clay, and the bristles were stimulated with a thin hair as above. Recorded responses were then analyzed in frame-to-frame mode.

Behavioral quantification. To quantify the cleaning reflex and its modification by experience, the following set-up was constructed (Fig. 3): A fly was suspended from a micromanipulator (M2, Narashige, Japan) by a metal microelectrode (25-10-1, FHC, New-Brunswick). Two channels capable of delivering controlled air puffs were used for mechanical stimulation. Each channel consisted of a positive pressure source (from a central pressure pump), delivering air regulated to a flow of $63 \mathrm{ml} / \mathrm{sec}$ by a flowmeter (Flowrator, Lab-Crest, Warminster, PA). The stimuli were delivered through capillaries (50 $\mu \mathrm{m}$ tip diameter) situated at a distance of approximately $0.5 \mathrm{~cm}$ from the fly. The direction of flow was regulated by 3-way air valves $(1-22-900$, General Valve, Fairfield, NJ). The valves were controlled by a pulse generator (Grass S88, Quincy, $\mathrm{MA}$, via an electronic interface that converted the square pulse to a biphasic square pulse. Each individual air puff was generated by a 5 msec electric pulse, and the air flow had an approximate duration of $300 \mathrm{msec}$. One channel was usually driven at $0.2 \mathrm{pulses} / \mathrm{sec}$ (pps) with the puff directed to the legulars, antero-noto-pleural, upper humeral, and lower humeral bristles; it was denoted the habituating channel (see below). The other channel was driven by train pulses ( $1 \mathrm{sec} \operatorname{train} / 3 \mathrm{sec}$ ) of $20 \mathrm{pps}$ directed to the dorsocentral area of the notum, stimulating the dorsocentral macrochaetae and microchaetae; this was denoted the dishabituating channel (see below). The response to each stimulus delivered by the habituating channel was observed under a stereomicroscope and quantified by recording the movement of the leg to landmark bristles, as detailed under Results.

Data analysis. Unless otherwise indicated, data are presented as means \pm SEM. Two-tailed Student's $t$ test was used for determination of statistical significance.

\section{Results}

Stimulation of thoracic bristles elicits a cleaning response in the legs

Flies respond to mechanical stimulation of the thoracic bristles by sweeping the stimulated area with the first or third leg, depending on the identity of the stimulated bristle(s). At the first stage of our study, we have determined the sensory fields of the thoracic macrochaetae. When the mechanical stimulus is directed at the following individual bristles, located in the anterior lateral part of the thorax, cleaning of this area is performed by the ipsilateral foreleg (Fig. 1): upper-humeral (UH), lower-humeral (LH), antero-noto-pleural (ANP), postero-noto-pleural (PNP), and tegulars (TEs). Stimulation of the following individual bristles elicits a cleaning response in the ipsilateral third leg: pre-sutural (PS), antero-supra-alar (ASA), postero-supraalar (PSA), antero-dorso-central (ADC), postero-dorso-central (PDC), antero-post-alar (APA), postero-post-alar (PPA), anteroscutellar (ASC), and postero-scutellar (PSC). Stimulation of the microchaetae covering the notum elicits a response in the third leg. A strong stimulus applied to the dorsocentral area of the notum, including the dorsocentral macro- and microchaetae, can also evoke a response in the first leg, which is weaker than the response in the third leg.

By observing the response to 20 consecutive stimuli (1 stimulus $/ 10 \mathrm{sec}$ ) delivered at each one of the aforementioned bristles, we obtained a probability of eliciting a leg response by stimulation of a specific bristle. We found that the probability of response elicited by single stimulation of an individual macrochaeta is always $<1.0$, and for several bristles is $\ll 1.0$. The highest probability of response was observed in the first leg upon stimulation of the ipsilateral tegula $(p=0.81 \pm 0.02, n=58)$. Probability of response of the third leg upon stimulation of the appropriate macrochaetae was lower than that observed in the first leg following stimulation of the appropriate macrochaetae. 

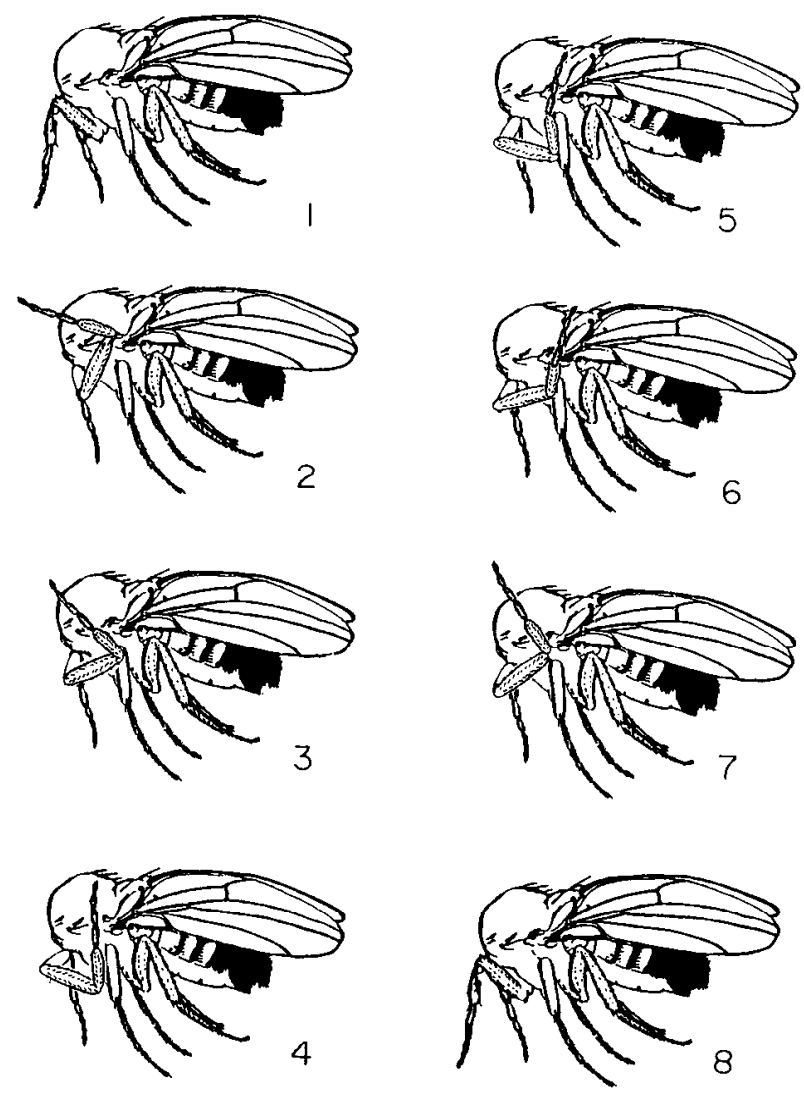

Figure 2. Sequence of movements performed by a decapitated Drosophila upon mechanical stimulation of the tegular bristles: 1 , before stimulation; $2-7$, response; 8 , return to resting position.

The highest probability of response in the third leg was detected upon stimulation of the PPA $(p=0.11 \pm 0.03, n=54)$. Nevertheless, stimulation of a field of dorsocentral microchaetae elicited frequent responses in the third leg. The relationship between bristle stimulation and leg response is in accordance with the results reported by Vandervorst and Ghysen (1980). Since probability of response of the foreleg was close to 1.0 upon simultaneous stimulation of the TEs, ANP, and both humerals (HUs), the cleaning response elicited upon stimulation of the latter group of bristles was chosen for further analysis.

\section{The cleaning response is a characteristic set of movements}

The cleaning response elicited in the foreleg by mechanical stimulation of the tegular bristles consists of a patterned set of movements executed by the leg. The entire behavioral act in which the tarsus sweeps the stimulated area is completed within approximately $1 \sec$ (Fig. 2). Usually this movement includes the cleaning of the whole area, arriving at the TEs. However, sometimes a partial response is observed, in which the leg cleans only the area anterior to the TEs (and see below).

\section{The cleaning response is modified by experience}

In preliminary experiments we found that following repeated monotonous stimulation, the cleaning response decreased and finally vanished. We have also often observed that when only part of the bristles that evoked response in the first leg were stimulated until the response vanished, stimulation of other, previously nonstimulated bristles, still elicited the response.

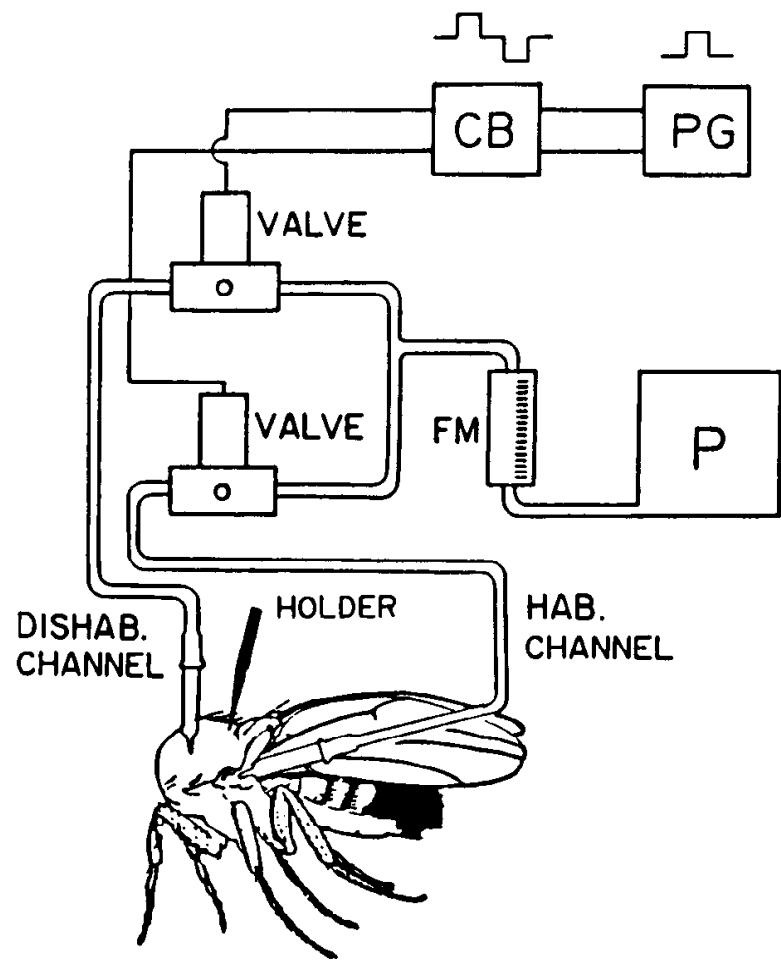

Figure 3. Schematic drawing of the experimental setup used to quantify the cleaning response: $P$, pressure source; $F M$, flow meter; $P G$, pulse generator; $C B$, electronic control box; VALVE, 3-way solenoid valves, HOLDER, metal microelectrode. Also depicted are the habituating $(H A B$.$) and dishabituating (DISHAB.) channels, delivering air puffs at$ the appropriate bristles.

These results suggested that response decrement was not due to motor fatigue and left open the possibility of habituation. We have therefore set out to quantify the modification of response by experience and to determine its characteristics.

We submitted flies to repetitive stimulation at $0.2 \mathrm{pps}$ by the channel denoted the habituating channel (see Materials and Methods), until the flies stopped responding to 10 consecutive stimuli. We defined such repetitive stimulation as a training session. We quantified the response of a fly in a training session by using 2 parameters. One was the magnitude of response to each stimulus. This was obtained by observing the foreleg and recording its movement on a scale of $0-4$. No response was denoted as 0 . Values from 1 to 4 were assigned to movement of the leg as follows: 1, tarsus stopped short of HUs; 2, tarsus reached the HUs and stopped therc; 3, tarsus reached the ANP and stopped there; 4 , tarsus arrived at the TEs, thus cleaning the whole stimulated area. The other parameter was the number of stimuli required for the fly to stop responding. This was taken as the number of stimuli delivered in a training session excluding the last 10 to which the fly did not respond. This parameter was denoted SSR (i.e., stimuli to stop responding).

Quantitative analysis of the response necessitated establishment of operational standards. This was because in preliminary experiments, we had observed variability in the behavior of decapitated flies which might be due to traumatic effects following anesthesia and surgery. Thus, of all recovered wild-type flies ( $n=183$ ), $31 \%$ seemed to be hyperexcited, moved the legs without stimulation, responded to bristle stimulation with the second leg, and/or continuously buzzed their wings. Following an initial screen we have therefore defined a set of criteria to 


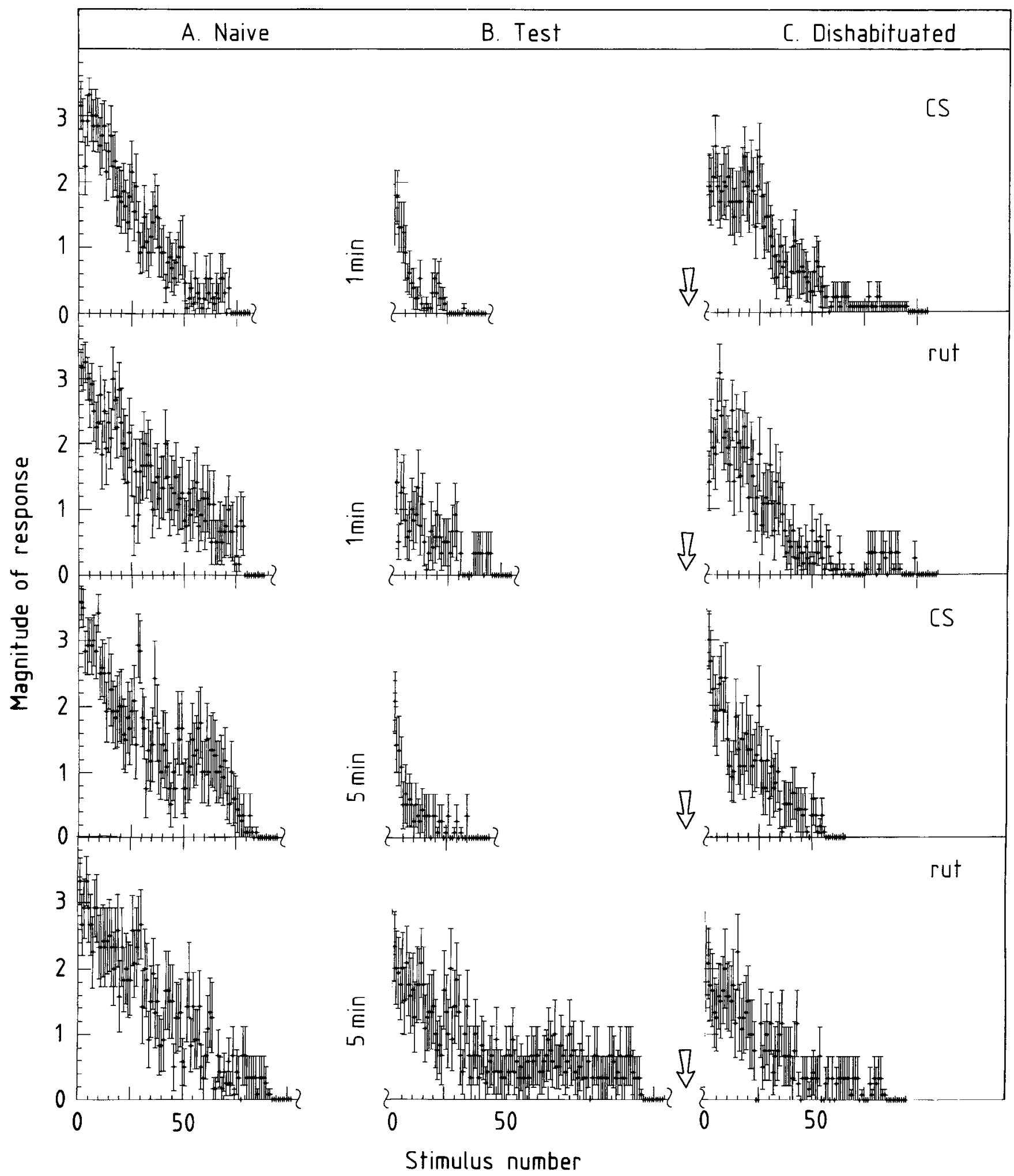

Figure 4. Decrement in the response of Drosophila to repeated thoracic bristles stimulation by the habituating channel during the first training session $(A)$, during a test following 1 or 5 min rest $(B)$, and following dishabituation $(C)$. During training and testing, air puffs were delivered at $0.2 \mathrm{pulses} / \mathrm{sec}$ as described in Materials and Methods. For the experimental set up, see Figure 3 . The magnitude of response was measured by observing the foreleg and noting its movement to landmark bristles, as detailed under Results. No response was denoted as $0,1-3$ are partial responses as judged by the distance travelled by the leg, and 4 is full response. Dishabituation stimuli, denoted by arrow, were delivered as described in Materials and Methods, $30 \mathrm{sec}$ after the last stimulus presented in the test. Each point is the mean \pm SEM of the responses of all the files tested. 


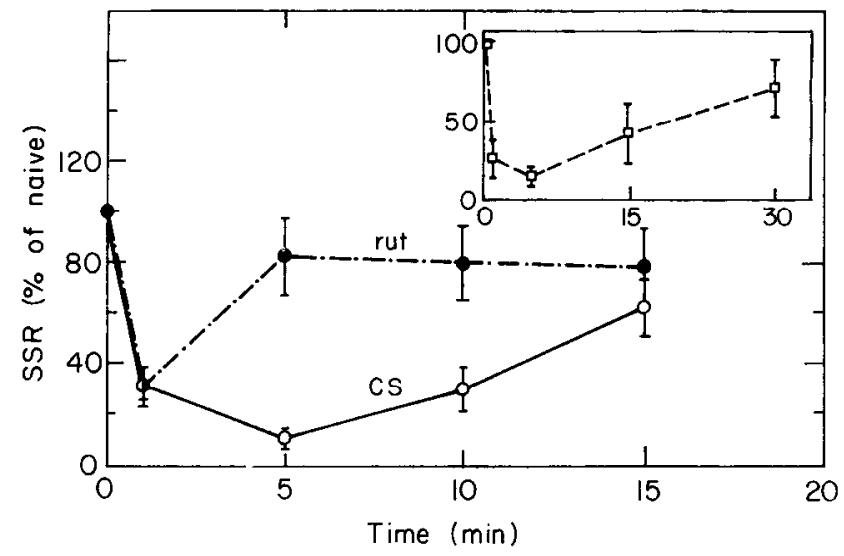

Figure 5. Time-dependent recovery of the response to bristle stimulation of habituated CS and rut flies, quantified in terms of SSR. Flies were trained once at $t=\mathrm{O}$, and then retrained (hence tested for their SSR) after the appropriate rest. Results are expressed as the percentage SSR (SSR of naive fly $=100 \%$ ). Open circles, CS flies, tested only at the indicated time point; closed circles, rut flies, tested only at the indicated time point. The SSR of rut significantly differs from that of CS at 5 and $10 \mathrm{~min}(p<0.001$ and $<0.02$, respectively). Inset, CS flies, tested consecutively at all time points (i.e., at 1, 5, 15, $30 \mathrm{~min}$ ).

permit reproducible quantification of the cleaning response and its modification by experience. These criteria were considered to minimize artifacts due to trauma as well as damage caused on stage. Only flies that conformed to the following criteria were included in our analysis: (1) The fly responded to simultaneous stimulation of the TEs, ANP, and HUs only with the ipsilateral foreleg. (2) SSR was between 10 and 100 in the first training session. This criterion was established because preliminary experiments showed that flies that did not stop responding after 100 stimuli in the first training session, seldom stopped responding even after 300 stimuli and seemed, therefore, permanently excited. (3) Following restoration of response by the dishabituating channel, the fly again ceased responding to the habituating channel, with an SSR of 10-200, which should also be between $15-200 \%$ of the SSR of the same fly in its first training session.

Of all the wild-type flies that showed response decrement upon repeated stimulation $(n=126), 41 \%$ fulfilled all the aforementioned criteria. An additional $32 \%$ displayed response decrement following repeated stimulation and restoration of response following stimulation by the dishabituating channel but did not fulfill all the numerical requirements stated above, especially in criterion 3 . An additional $27 \%$ did not display recovery of response following presentation of the dishabituating stimulus.

The response of wild-type flies that conformed to the aforementioned criteria in their first training session is depicted in Figure $4 A$. The SSR of these flies was $46.5 \pm 2.9$.

\section{Response decrement conforms to parametric characteristics of habituation}

To assess whether the aforementioned decrement and disappearance of reflexive response should be considered habituation, the behavior was analyzed using the parametric characteristics established by Thompson and Spencer (1966).

1. Time-dependent spontaneous recovery. We measured the magnitude of individual responses and SSR after a period of rest following the first training session (Figs. $4 B$ and 5). Response

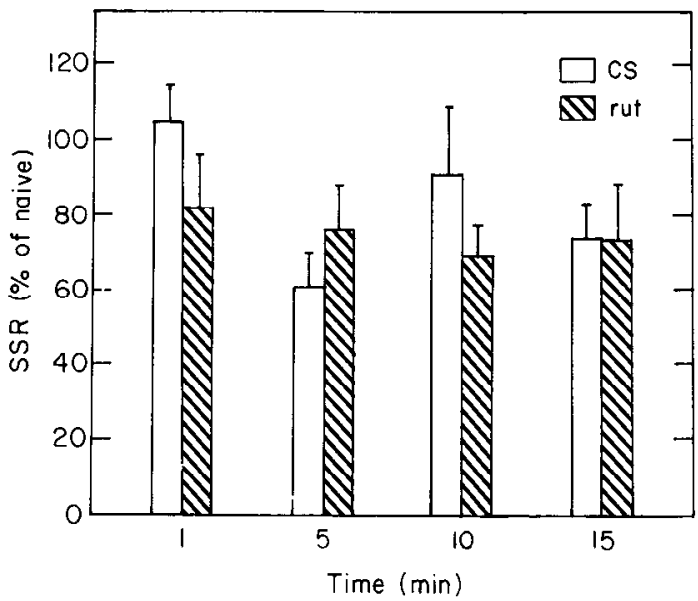

Figure 6. Dishabituation of response to bristle stimulation in CS and rut flies. Data are presented as percentage SSR (SSR of naive fly $=$ $100 \%$ ). Flies were trained once, retrained following rest of $1,5,10$, or $15 \mathrm{~min}$, and following cessation of response, were immediately dishabituated and retested for SSR.

decrement was most pronounced after $5 \mathrm{~min}$; the response then gradually recovered (Fig. 5).

2. Dishabituation. As already mentioned, when a fly, after being trained, was immediately stimulated with the dishabituating channel, it showed recovery of response (SSR was $82.4 \pm$ $5.9 \%$ of the naive fly value). Results for dishabituation following retraining at various time points after the first training session are depicted in Figures $4 C$ and 6.

3. Stimulus frequency dependence. When the frequency of stimulation was increased by a factor of 5 (to $1 \mathrm{pps}$ ), the flies were habituated within a much shorter time $(207 \pm 10 \mathrm{sec}$ for $0.2 \mathrm{pps}, 46 \pm 7 \mathrm{sec}$ for $1 \mathrm{pps})$. The SSR was not significantly different on comparing the 2 frequencies of stimulation (46.5 \pm 6.5 for 1 pps, $42.5 \pm 2$ for $0.2 \mathrm{pps}$ ).

4. Stimulus intensity dependence. When air flow was reduced by half, the SSR of naive flies decreased to $23.1 \pm 4.5$.

Potentiation of habituation was not directly tested in this study, but its existence was suggested by the difference observed between the SSR of groups of flies trained in 2 different ways. Some flies were subjected to a training procedure in which each fly was tested for habituation, dishabituation, and then for response recovery after 4 consecutive resting intervals of $1,5,15$, and $30 \mathrm{~min}$. This training procedure thus consisted of habituation, dishabituation, and habituation, rest for $1 \mathrm{~min}$, again habituation, rest for $5 \mathrm{~min}$ and habituation, and so on. The SSR after $15 \mathrm{~min}$ rest in flies that had only 2 habituating sessions (i.e., the usual paradigm) tended to be larger than in flies that were habituated 5 consecutive times (i.e., the paradigm described here; inset in Fig. 5).

Taken together, our results demonstrate that the modification by experience of the cleaning response conforms to parametric criteria set for habituation (Thompson and Spencer, 1966). Habituation of dishabituation and below-zero habituation were not tested in our system. Testing of generalization, which is not detected in various systems (e.g., Pinsker et al., 1970), is not feasible in the present paradigm. We have also determined that habituation is confined to the field that has received the habituating stimulus: When the stimulus to a fly that has just habituated was moved to new bristles, the fly responded as if nonhabituated. For example, following decrement of response 
to stimulation of the TEs only, response in the same leg was still elicited by stimulation of the ANP and HUs. We have also tested the specificity of habituation as a function of stimulus frequency: When a fly was habituated to a stimulus of a certain frequency, it was also nonresponsive to stimuli below that frequency but recovered responsiveness if stimulated with a frequency 5-10 times higher (data not shown).

\section{Reflex modification is abnormal in the mutant rut}

We have tested habituation, time-dependent recovery of response, and dishabituation in the mutant rut, which is defective in learning (Duerr and Quinn, 1982; Dudai, 1983; Livingstone et al., 1984) and in adenylate cyclase activity (Dudai et al., 1983, 1984; Livingstone et al., 1984). Naive rut flies showed decrement and disappearance of the cleaning response after repeated presentation of the mechanical stimulus (Fig. $4 A$ ). The fraction of flies showing decrement and restoration of response and conforming to all the quantitative criteria set by us above was $34 \%$. In addition, $49 \%$ of $r u t$ flies showed decrement and restoration of response following the aforementioned procedures but did not fulfill all the numerical requirements of the criteria. An additional $17 \%$ did not respond after presentation of the dishabituating stimulus.

SSR in naive rut flies that fulfilled the criteria $(52.3 \pm 3.6)$, was slightly, but not significantly, higher than that of CS. After 1 min rest, habituation was clearly apparent (Fig. $4 B$ ), and the SSR was identical to that displayed by CS (Fig. 5). However, already after $5 \mathrm{~min}$ of rest, rut response increased to a plateau value close to that observed in the naive fly both in terms of the magnitude of response (Fig. $4 B$ ) and SSR (Fig. 5). After 10 min rest, the response in CS was still significantly different from the response of rut flies. The rut mutants thus behaved as though having a fleeting memory. When dishabituated immediately after training, SSR in rut recovered to $74.9 \pm 6.0 \%$ of the naive fly value, not significantly different from CS (Fig. 6).

\section{Discussion}

Studies of single-gene mutations in Drosophila may contribute to our understanding of elementary mechanisms of learning and memory (Aceves-Piña et al., 1983; Dudai, 1986). Several singlegene mutations that affect learning and memory have been isolated among the progeny of chemically mutagenized flies. Other, existing mutations were also found to exert relatively specific effects on learning and memory in different conditioning paradigms (reviewed as above). In several cases, the affected gene product has been identified, and in 2 cases the gene has been cloned (Scholnick et al., 1983; Chen et al., 1986). The genes thus cloned have proven to be complex and developmentally regulated. Thus, genetic analysis combined with molecular biological studies and with fine behavioral analysis, make it possible, in principle, to elucidate the contribution of specific macromolecules to learning and memory systems.

Until now, a major disadvantage of the neurogenetic dissection of learning in Drosophila has been the lack of data on the effect of learning mutations at the cellular level. The Drosophila CNS is dense and compact, making the identification of cellular components involved in behavioral response very difficult. In addition, most learning paradigms used in Drosophila to date involve many complex sensory and motor systems. Even when what might be considered a relatively simple reflex, the proboscis-extension response, was studied (Duerr and Quinn, 1982), the experimental system was highly complex. The sensory neu- rons innervating the tarsal chemosensory bristles are heterogeneous in their sensory specificity and may also respond to mechanical stimulation (Wolbarsht and Dethier, 1958), and single neurons are not practically identifiable (Nayak and Singh, 1983).

We have, therefore, set out to identify a learning paradigm that might be better suited to at least partial analysis of some cellular correlates of learning in Drosophila. The cleaning reflex offers advantages in this respect. It is elicited by hairs invariably positioned on the cuticle, each one innervated by a single sensory neuron which is sensitive only to mechanical stimulus and has an essentially invariant projection to the thoracic ganglion. Moreover, the mechanical stimulus is easily controlled.

Cleaning reflexes are ubiquitous in the animal kingdom and are composed of a fixed action pattern of limb movement, which is often astonishingly similar in phylogenetically remote species (e.g., Franzisket, 1963). In Drosophila, the cleaning reflex following tactile stimulus to a bristle consists of a vigorous and rapid movement towards the affected bristle(s). The identity of the leg in which the reflex is elicited is a function of the stimulated bristle. Our preliminary observations indicated that the fly may adjust under certain conditions its response on the basis of experience. This was indeed confirmed by systematic analysis. The overall decrease in response observed with repeated monotonous stimulation fulfilled parametric criteria expected from habituation (Thompson and Spencer, 1966), which is the most elementary form of learning.

Most interestingly, the mutant rut, deficient in acquisition and short-term memory in several associative learning paradigms (Aceves-Piña et al., 1983; Dudai et al., 1984; Livingstone et al., 1984), displayed an abnormally rapid diminution of habituation. This is compatible with the behavior of rut in other learning paradigms (Dudai, 1983; Dudai et al., 1984; Tully and Quinn, 1985. The data also corroborate the conclusion that the modification of the cleaning response is, indeed, a form of behavioral plasticity. In addition, they are in accordance with the hypothesis that habituation and sensitization share mechanisms with associative learning (Kandel and Schwartz, 1982). This was also the conclusion reached by Duerr and Quinn (1982), who reported that proboscis-extension reflex, elicited by a chemical stimulus, habituates less and sensitizes for a shorter period in rut compared with normal flies. It thus seems that some properties of adenylate cyclase that are defective in rut, e.g., activation by $\mathrm{Ca}^{2+}$-calmodulin, play an elementary role in the early phases of memory formation in both nonassociative and associative learning tasks, involving different sensory modalities.

It should, however, be noted that rut can habituate and dishabituate; the normal counterpart of its defective gene product is therefore only one of the components playing a role in early memory formation, and other processes, not requiring normal activity of this gene product, operate in parallel. In the present paradigm the role of $\mathrm{rut}^{+}$gene product appears to be especially (but not exclusively) important during early memory, rather than during acquisition. The relative weight of the role of $r u t^{+}$ gene product in acquisition and short-term memory phases may depend on the nature of the learning paradigm (Dudai, 1986).

The input system involved in the cleaning reflex offers advantages not only for behavioral but also for neuroanatomical and certain physiological investigations. The sensory neuron can be traced by backfilling with HRP or other markers (Ghysen, 1980; Burg and $\mathrm{Wu}, 1986)$. Fine morphology of the axon, in- 
cluding axonal branching and axonal varicosities in the CNS, can be discerned (Corfas, unpublished observations). The morphology can be also analyzed at the electron microscopic level, including both dendritic (Burg and $\mathrm{Wu}, 1986$ ) and axonal ultrastructure (Corfas, unpublished observations). It is also possible to record the receptor potential and the action potentials generated in the sensory neurons upon deflection of the hairs with extracellular electrodes (Corfas and Dudai, 1988). Analysis of the sensory neurons at these levels should dissect the role of these neurons in the experience-dependent modification of the reflex. It is of relevance to note that sensory components of modifiable reflexes were indeed shown to be sites of plasticity in other organisms (Kandel and Schwartz, 1982; Alkon, 1984; Byrne, 1985). Cellular components of the output system involved in the cleaning reflex are also approachable. Motoneurons that execute the cleaning reflex in the leg can be identified by anatomical techniques (Green, 1981). In addition, spontaneous and evoked potentials can be recorded from the leg (Dudai et al., 1987).

Thus, although not all the neurons involved in the cleaning reflex are as yet accessible to electrophysiological manipulations, and the interneurons have not yet been identified, some potentially important and relevant cellular facets of 2 of the main components that mediate the reflex can be studied. These aspects include fine neuronal morphology, i.e., ultrastructural correlates of central connectivity, which was shown to be altered by experience in both invertebrates (Bailey and Chen, 1983, 1985) and vertebrates (Horn ct al., 1985). Elcctrical and pharmacological studies of both the sensory and motor neurons, which are feasible in this system, might also provide insight into the role of discrete gene products in neuronal development and plasticity.

\section{References}

Aceves-Piña, E. O., R. Booker, J. S. Duerr, M. S. Livingstone, W. G. Quinn, R. F. Smith, P. P. Sziber, B. I. Tempel, and T. P. Tully (1983) Learning and memory in Drosophila, studied with mutants. Cold Spring Harbor Symp. Quant. Biol. 48: 831-840.

Alkon, D. L. (1984) Calcium-mediated reduction of ionic currents: A biophysical memory trace. Science 226: 1037-1045.

Bailey, C. H., and M. Chen (1983) Morphological basis of long-term habituation and sensitization in Aplysia. Science 220: 91-93.

Bailey, C. H., and M. Chen (1985) Morphological basis of short-term habituation in Aplysia. Soc. Neurosci. Abstr. 11: 1110.

Burg, M. G., and C-F. Wu (1986) Differentiation and central projection of peripheral sensory cells with action potential block in Drosophila mosaics. J. Neurosci. 6: 2968-2970.

Byrne, J. H. (1985) Neural and molecular mechanisms underlying information storage in Aplysia: Implications for learning and memory. Trends Neurosci. 9: 478-482.

Carew, T. J., and C. L. Sahley (1986) Invertebrate learning and memory: From behavior to molecules. Annu. Rev. Neurosci. 9: 435-487.

Chen, C. N., S. Denome, and R. L. Davis (1986) Molecular analysis of $\mathrm{CDNA}$ clones and the corresponding genomic coding sequences of the Drosophila dunce gene, the structural gene for cAMP phosphodiesterase. Proc. Natl. Acad. Sci. USA 83: 9313-9317.

Corfas, G., and Y. Dudai (1988) Sensory adaptation of neurons innervating thoracic bristles of Drosophila melanogaster. Soc. Neuroci. Abstr. 13: 1164.

Dudai, Y. (1977) Properties of learning and memory in Drosophila melanogaster. J. Comp. Physiol. 114: 69-89.

Dudai, Y. (1983) Mutations affect storage and use of memory differentially in Drosophila. Proc. Natl. Acad. Sci. USA 80: 5445-5448.

Dudai, Y. (1986) Cyclic AMP and learning in Drosophila. Adv. Cyclic Nucleotide Protein Phosphoryl. Res. 20: 343-361.
Dudai, Y. (1987) The cAMP cascade in the nervous system: Molecular sites of action and possible relevance to neuronal plasticity. CRC Crit. Rev. Biochem. 22: 221-281.

Dudai, Y., A. Uzzan, and S.Zvi (1983) Abnormal activity of adenylate cyclase in the Drosophila memory mutant rutabaga. Neurosci. Lett. 42: 207-212.

Dudai, Y., S.Zvi, and S. Segel (1984) A defective conditioned behavior and a defective adenylate cyclase in the Drosophila mutant rutabaga. J. Comp. Physiol. 155: 569-576.

Dudai, Y., J. Buxbaum, G. Corfas, and M. Ofarim (1987) Formamidines interact with Drosophila octopamine receptors, alter the flies' behavior and reduce learning ability. J. Comp. Physiol. 161: 739746.

Duerr, J. S., and W. G. Quinn (1982) Three Drosophila mutations that block associative learning also affect habituation and sensitization. Proc. Natl. Acad. Sci. USA 79: 3646-3650.

Fischbach, K. F. (1981) Habituation and sensitization of the landing response of Drosophila melanogaster. Naturewiss. 68: 332.

Franzisket, L. (1963) Characteristics of instinctive behavior and learning in reflex activity of the frog. Anim. Behav. 11: 318-324.

Ghysen, A. (1980) The projection of sensory neurons in the central nervous system of Drosophila: Choice of the appropriate pathway. Dev. Biol. 78: 521-541.

Goelet, P., V. F. Castellucci, S. Schacher, and E. R. Kandel (1986) The long and the short of long-term memory-A molecular framework. Nature 322: 419-422.

Green, S. H. (1981) Segment-specific organization of leg motoneurones is transformed in bithorax mutants of Drosophila. Nature 286: 6567.

Hall, J. C. (1984) Complex brain and behavioral functions disrupted by mutations in Drosophila. Dev. Genet. 4: 355-378.

Horn, G., P. Bradley, and B. J. McCabe (1985) Changes in the structure of synapses associated with learning. J. Neurosci. 5: 3161-3168.

Kandel, E. R., and J. H. Schwartz (1982) Molecular biology of learning: Modulation of transmitter release. Science 218: 433-443.

Lewis, E. G. (1960) A new standard food medium. Drosophila Inf. Ser. 34: $117-118$.

Livingstone, M. S., P. P. Sziber, and W. G. Quinn (1984) Loss of calcium/calmodulin responsiveness in adenylate cyclase of rutabaga, a Drosophila learning mutant. Cell 37: 205-215.

Mariath, H. A. (1985) Operant conditioning in Drosophila melanogaster wild-type and learning mutants with defects in the cyclic AMP metabolism. J. Insect Physiol. 31: 779-787.

Menne, D., and H. C. Spatz (1977) Colour vision in Drosophila melanogaster. J. Comp. Physiol. 114: 301-312.

Nayak, S. V., and R. N. Singh (1983) Sensilla on the tarsal segments and mouthparts of adult Drosophila melanogaster Meigen. Int. J. Insect Morphol. Embryol. 12: 273-291.

Nestler, E. J., and P. Greengard (1984) Protein Phosphorylation in the Nervous System, Wiley, New York.

Pinsker, H., I. Kupfermann, V. Castellucci, and E. R. Kandel (1970) Habituation and dishabituation of the gill-withdrawal reflex in Aplysia. Science 167: 1740-1742.

Power, M. E. (1948) The thoracico-abdominal nervous system of an adult insect, Drosophila melanogaster. J. Comp. Neurol. 88: 347-409.

Quinn, W. G. (1984) Work in invertebrates on the mechanisms underlying learning. In Biology of Learning, P. Marler and H. S. Terace, eds., pp. 197-246, Springer-Verlag, Berlin.

Quinn, W. G., W. A. Harris, and S. Benzer (1974) Conditioned behavior in Drosophila melanogaster. Proc. Natl. Acad. Sci. USA 71: $708-712$.

Scholnick, S. B., B. A. Morgan, and J. Hirsh (1983) The cloned dopa decarboxylase gene is developmentally regulated when reintegrated into the Drosophila genome. Cell 34: 37-45.

Thompson, R. F., and W. A. Spencer (1966) Habituation: A model phenomenon for the study of neuronal substrates of behavior. Psychol. Rev. 73: 16-43.

Tully, T., and W. G. Quinn (1985) Classical conditioning and retention in normal and mutant Drosophila melanogaster. J. Comp. Physiol. 157: 263-277.

Vandervorst, P., and A. Ghysen (1980) Genetic control of sensory connections in Drosophila. Nature 286: 65-67.

Wolbarsht, M. L., and V. G. Dethier (1958) Electrical activity in the chemoreceptors of the blowfly. J. Gen. Physiol. 42: 393-412. 